\title{
Ethics of Location-Based Data in Crisis Situations
}

\author{
Alan M. MacEachren ${ }^{\text {a, * }}$ \\ ${ }^{a}$ Department of Geography, Pennsylvania State University, maceachren@psu.edu \\ * Corresponding author
}

Keywords: Location-based Data, VGI, Remote Sensing, Humanitarian Relief, Crisis Management

\begin{abstract}
:
This presentation will provide an overview of a Workshop-based effort on ethics in location-based, organized by the Scientific Responsibility, Human Rights, and Law Program of the American Association for the Advancement of Science (AAAS). More specifically, the AAAS organized three workshops during 2017 and 2018 directed to exploring the ethical implications of collecting, analysing, and acting upon location-based data in crisis situations -- "Developing Ethical Guidelines and Best Practices for the Use of Volunteered Geographic Information and Remotely Sensed Imagery in Crisis Situations.". The outcome of those workshops and follow up efforts was a document detailing principles and guidelines with the objective of empowering crisis response actors to use location-based data responsibly and ethically.

On behalf of all those involved, as a Participant in all three workshops and a AAAS Fellow, I will present an overview of the results of this effort. The presentation will outline the five principles developed and provide examples of their motivation and use:
\end{abstract}

1. Do No Harm: Identify and minimize potential risk, particularly as they may affect the vulnerability of individuals and populations

2. Define Your Purpose: Ensure action is mission-driven and goal-oriented

3. Do Good Science: Employ scientifically rigorous and responsible methods

4. Collaborate and Consult: Engage with local partners

5. Give Access to Your Data: Share data openly, when safe and practicable

The presentation will also reflect on (a) the specific relevance of this effort and its outcome for the international cartographic community and (b) our obligation as academic/professional cartographers to address the dual challenges of leveraging locational data cartographically to support crisis management and humanitarian efforts while also guarding against misuse of the data collected and map generated. I will conclude by reflecting on my experience in working with a diverse, interdisciplinary, international group on this hard problem. 